\title{
Wide-Angle Quasar Feedback
}

\section{George Chartas and Sarah Strickland}

Department of Physics and Astronomy, College of Charleston, Charleston, SC, 29424, USA

email: chartasg@cofc.edu

\begin{abstract}
We present results from the detection of relativistic winds launched near the innermost stable circular orbits of supermassive black holes. A recent detection of a powerful wind in the X-ray-bright narrow absorption line (NAL) $z=1.51$ quasar HS $0810+2554$ strengthens the case that quasars play a significant role in feedback. In both deep Chandra and XMM-Newton observations of HS 0810 we detected blueshifted absorption lines implying outflowing velocities ranging from $0.1 c$ and $0.4 c$. The presence of both an emission line at $6.8 \mathrm{keV}$ and an absorption line at $7.8 \mathrm{keV}$ in the spectral line profile of HS 0810 is a characteristic feature of a P-Cygni profile supporting the presence of an expanding outflowing highly ionized Fe absorber. A hard excess component is detected in the XMM-Newton observation of HS 0810 possibly originating from reflection off the disk. Modelling of the XMM-Newton spectrum constrains the inclination angle to be $<35^{\circ}$ (68\% confidence). The presence of relativistic winds in both low inclination angle NAL quasars as well as in high inclination angle BAL quasars implies that the solid angle of quasar winds may be quite large. The larger solid angle of quasar winds would also indicate that their contribution to the regulation of the host galaxy may be more important than previously thought.
\end{abstract}

Keywords. galaxies: formation — quasars: absorption lines - X-rays: galaxies —intergalactic medium

\section{Summary of Results}

We summarize the results from the latest X-ray observations of HS 0810 as follows:

1. A highly ionized, relativistic, and variable wind is present in both the $100 \mathrm{ks}$ Chandra and $46 \mathrm{ks} X M M$-Newton observations of HS 0810 . We find the ionization parameter of the $\mathrm{X}$-ray outflowing absorber to lie in the range $\log \left(\xi / \mathrm{erg}_{\mathrm{cm} \mathrm{s}}{ }^{-1}\right)=3.2-3.5$, the hydrogen column density to lie within the range $N_{\text {Habs }}=1.7-3 \times 10^{23} \mathrm{~cm}^{-2}$, and the outflow velocity to vary within the range $v_{\text {abs }}=0.1-0.4 \mathrm{c}$.

2. Our interpretation of the relativistic nature of the outflow is consistent with the detection of a SiXIV absorption line blueshifted by the same amount as the highly ionized Fe line in the $100 \mathrm{ks}$ Chandra spectrum of image A+B. The presence of an outflow is also supported by the detection of a P-Cygni profile in the $100 \mathrm{ks}$ Chandra spectrum of image $\mathrm{A}+\mathrm{B}$. We constrain the covering factor of the relativistic wind to be $f_{\mathrm{c}}=0.6_{-0.3}^{+0.2}$.

3. By modelling the hard X-ray excess detected in the XMM-Newton spectrum of HS 0810 as blurred relativistic reflection from the accretion disk we constrain the inclination angle of the disk to be $<35^{\circ}$ ( $68 \%$ confidence). Such a small angle is consistent with models that posit NAL quasars as objects observed at relative low inclination angles.

4. The mass-outflow rate of HS 0810 is found to lie in the range of $\dot{M}=1.5-2.1 \mathrm{M}_{\odot} y r^{-1}$ and is comparable to the accretion rate $1 \mathrm{M}_{\odot} y r^{-1}$ (assuming an accretion efficiency of 0.1 and an Eddington ratio of 0.1 ).

5. The Chandra spectra of images $\mathrm{C}$ and $\mathrm{A}+\mathrm{B}$ differ significantly. We propose as a possible explanation for this difference a microlensing event occurring in image C. This interpretation is consistent with the anomalous X-ray flux ratio of image $\mathrm{C}$. 